\title{
Qualitative Analysis of Factors Influencing Patient Persistence and Adherence to Prescribed Overactive Bladder Medication in UK Primary Care
}

\author{
Mahmood Ali (D) - Sarah Grogan - Sue Powell - Leanne Staniford • \\ Jameel Nazir · Margarita Landeira · Patrick J. O. Covernton • \\ Ashley Jaggi · Francis Fatoye · Maxine Holt
}

Received: June 18, 2019 / Published online: September 26, 2019

(C) The Author(s) 2019

\begin{abstract}
Introduction: Pharmacotherapy for overactive bladder $(\mathrm{OAB})$ is generally associated with low rates of persistence and adherence. This study was conducted to explore the patient journey in a UK primary care setting (experiences, perceptions, attitudes, and levels of engagement and expectations) and identify self-reported reasons for patient non-adherence and/or non-persistence to medications for OAB.

Methods: This was a qualitative, non-interventional study involving one-to-one semi-structured, face-to-face or phone interviews with individuals aged 40-80 years, diagnosed with $\mathrm{OAB}$, and currently taking, or having taken, either antimuscarinic or $\beta_{3}$-adrenoceptor agonist medications within the last 12 months.
\end{abstract}

Enhanced Digital Features To view enhanced digital features for this article go to https://doi.org/10.6084/ m9.figshare.9810755.

Electronic Supplementary Material The online version of this article (https://doi.org/10.1007/s12325019-01098-y) contains supplementary material, which is available to authorized users.

M. Ali $(\bowtie) \cdot$ S. Grogan · S. Powell · L. Staniford .

A. Jaggi - F. Fatoye $\cdot$ M. Holt

Manchester Metropolitan University, Manchester,

UK

e-mail: mmd.ali01@gmail.com

J. Nazir · M. Landeira · P. J. O. Covernton Astellas Pharma Europe Ltd, Chertsey, UK
Thematic analyses of interview transcripts identified themes surrounding the participants' experiences with $\mathrm{OAB}$.

Results: A total of 20 interviews were conducted (face-to-face, $n=13$; telephone, $n=7$ ). Interviews from five men and 13 women (mean age 70 years) were included in the final analysis. The most common $\mathrm{OAB}$ symptoms reported included urgency, frequency, incontinence and nocturia. Several key themes of factors influencing persistence and/or adherence to prescribed $\mathrm{OAB}$ medication were identified: patients' attitude and condition adaptation behaviour; support with treatment; unmet efficacy/tolerability expectations; drug/condition hierarchy. Non-adherence and/or non-persistence to $\mathrm{OAB}$ medication was largely intentional, with patients balancing side effects against perceived clinical benefits. Perceived lack of efficacy was the primary reason for discontinuing treatment. Other factors cited included side effects of medication (either experienced or a fear of future effects), a general aversion to long-term medication taking, drug/condition hierarchy relative to other comorbidities, and limited healthcare professional (HCP) support/engagement. Patients expressed condition adaptation behaviours to help self-manage their condition.

Conclusion: Persistence and adherence to $\mathrm{OAB}$ medication may be suboptimal. HCPs might be able to improve persistence and adherence by fostering realistic treatment expectations and 
scheduling regular medication reviews. These measures may help optimise patient care and support more adherent behaviours, thus minimising the impact of undertreated $\mathrm{OAB}$ on patient quality of life.

Funding: Innovate UK and Astellas Pharma Europe Ltd (APEL).

Keywords: Adherence; Antimuscarinic agents; Drug/condition hierarchy; Mirabegron; Overactive bladder; Patients' attitude; Persistence; Qualitative research; Symptom adaptation behaviour; Unmet efficacy/tolerability expectations

\section{INTRODUCTION}

Overactive bladder (OAB) is a common, distressing and often chronic condition characterised by urinary urgency, usually accompanied by increased daytime frequency and/or nocturia, with or without urinary incontinence, in the absence of urinary tract infection or other detectable disease [1]. OAB is common in both men and women, with approximately 546 million adults worldwide (10.9\%) estimated to be affected by OAB in 2018 [2]. The prevalence of $\mathrm{OAB}$ increases with advancing age [3-5], and $\mathrm{OAB}$ is expected to become more common in the UK because of an increasingly ageing population [6]. OAB adversely affects health-related quality of life (HRQoL), including emotional, sexual and work productivity impacts [7, 8]. Individuals with $\mathrm{OAB}$ express anxiety, fear of incontinence, a sense of depression and hopelessness associated with their condition [9].

Behavioural and lifestyle modifications are the initial management strategies recommended for OAB [10]. Pharmacotherapy is recommended if symptom management through these conservative measures is ineffective. Pharmacotherapy includes antimuscarinic agents or the $\beta_{3}$-adrenoceptor agonist mirabegron (a second drug in this class is only available in Japan) $[10,11]$. As OAB can be chronic and is often progressive, patients may need to continue with treatment for lasting symptom relief [12].
Despite the considerable HRQoL impact of $\mathrm{OAB}$, individuals are often reluctant to consult a healthcare professional (HCP) about their condition $[9,13]$. Among those who seek treatment, low rates of persistence and/or adherence with pharmacotherapy are evident, especially for antimuscarinic agents. A systematic literature review of real-world data in $\mathrm{OAB}$ reported 1 -year persistence rates of $12-25 \%$ and $32-38 \%$ for antimuscarinic agents and mirabegron, respectively [14]. Median time to discontinuation was less than 5 months for antimuscarinic agents (except one study, 6.5 months [15]) and 5.6-7.4 months for mirabegron [14]. A UK Clinical Practice Research Datalink retrospective study [16] found that median time to discontinuation of antimuscarinic agents was 1.0-2.6 months compared with 5.6 months for mirabegron.

Non-adherence and/or non-persistence to pharmacological treatment has a major impact on health outcomes and healthcare resource utilisation, especially for long-term conditions such as OAB [7, 14, 17-21]. While a number of studies have explored persistence and/or adherence to OAB medications, the patients' perspectives and the reasons for discontinuing or not starting treatment have not been examined comprehensively. Identification of the reasons for non-adherence and/or non-persistence would provide insights to enable the development of measures to support long-term treatment goals. The objectives of this study were to explore patient treatment, experiences, perceptions, attitudes, and levels of engagement and expectations; and identify self-reported reasons for patient non-adherence and/or nonpersistence to medications for $\mathrm{OAB}$ in a $\mathrm{UK}$ primary care setting.

\section{METHODS}

\section{Study Design}

This was a qualitative, non-interventional study involving one-to-one interviews. Participants were required to meet the predetermined inclusion criteria: men or women aged 40-80 years; diagnosed with OAB; fluent in 
English; and currently taking, or having taken, either antimuscarinic agents or $\beta_{3}$-adrenoceptor agonists for $\mathrm{OAB}$ within the last 12 months. Individuals with memory impairment, cognitive decline or dementia (as documented within medical records) were not eligible for inclusion. Two UK National Health Service Research and Development sites (covering Kent, Surrey, and Sussex; and Greater Manchester) invited primary care practices (PCPs) from these regions to participate in this study. PCPs were sent the study protocol, copies of the patient information sheet, a study poster to display in the practice, and a research information sheet for practices form to help them decide whether they were able to participate in this study.

Participating PCPs compiled lists of their registered patients with $\mathrm{OAB}$ and applied the study inclusion and exclusion criteria. An HCP from each of the PCPs then reviewed the list of potential participants to confirm that those who progressed to be invited to participate in the study were appropriate to do so (correctly coded $\mathrm{OAB}$, not suffered recent bereavement/terminally ill, etc.). Invitation packs were posted to the home address of eligible participants. This included an information sheet outlining the area of research and rationale (Supplementary Appendix A). Participants who returned consent-to-be-contacted forms were telephoned by the study team to reiterate the purpose of the research, confirm their consent to take part in this study, and to arrange interviews. This study has gone through a health research authority and ethics review with NW Liverpool East REC (Central Manchester is the lead Research \& Development site). IRAS project ID 198502. All participants provided their written consent to take part in this study.

\section{Interviews}

Semi-structured interviews were conducted face-to-face or over the telephone by L.S., a research associate with more than 5 years' experience in qualitative research. A reflective journal recorded by the interviewer detailed that this was not a familiar topic of research, thus interviews were initiated with no preset assumptions or bias regarding medication persistence and/or adherence in relation to OAB. Participants were briefed on the interviewer's research experience and the purpose of the interviews prior to the interview commencing; they were also made aware that the interviewer had no commercial interests related to the study. Interviews were designed to take approximately $1 \mathrm{~h}$ and followed a discussion guide (see Supplementary Appendix B). They were conducted in participants' homes (Greater Manchester) and over the telephone (Kent, Surrey, and Sussex). Presence of non-participants (i.e. partners/family members) was at the discretion of the participants. Each interview was voice recorded and notes taken. Participants received a $£ 10$ store voucher upon interview completion. Participants had no further involvement following the interviews and repeat interviews were not performed.

The discussion guide was produced by academic researchers with expertise in qualitative research methods, with input from subject matter experts. Questions were framed to elicit full responses and obtain a narrative of the patient journey in their own words: starting with $\mathrm{OAB}$ history and coping strategies, interactions with their HCPs, treatment history, and treatment expectations; following up with a discussion of their strategies for adherence and finally the opportunity for any further comments (Supplementary Appendix B).

\section{Analyses}

Interviews were transcribed verbatim (by M.A.). A sample of transcripts (3/18 transcripts; $17 \%)$ was reviewed against corresponding recordings by the Chief Investigator (M.H.) to ensure completeness and accuracy of the transcripts. In addition, an academic Health Psychologist (S.G.), with more than 20 years' experience of conducting and publishing qualitative research, performed a validation crosscheck of the resulting themes.

Transcribed data were collectively analysed, using NVivo Pro 11 software (QSR International, Warrington, UK), to identify a set of themes on the basis of the method of thematic analysis by Braun and Clarke [22]. Data 


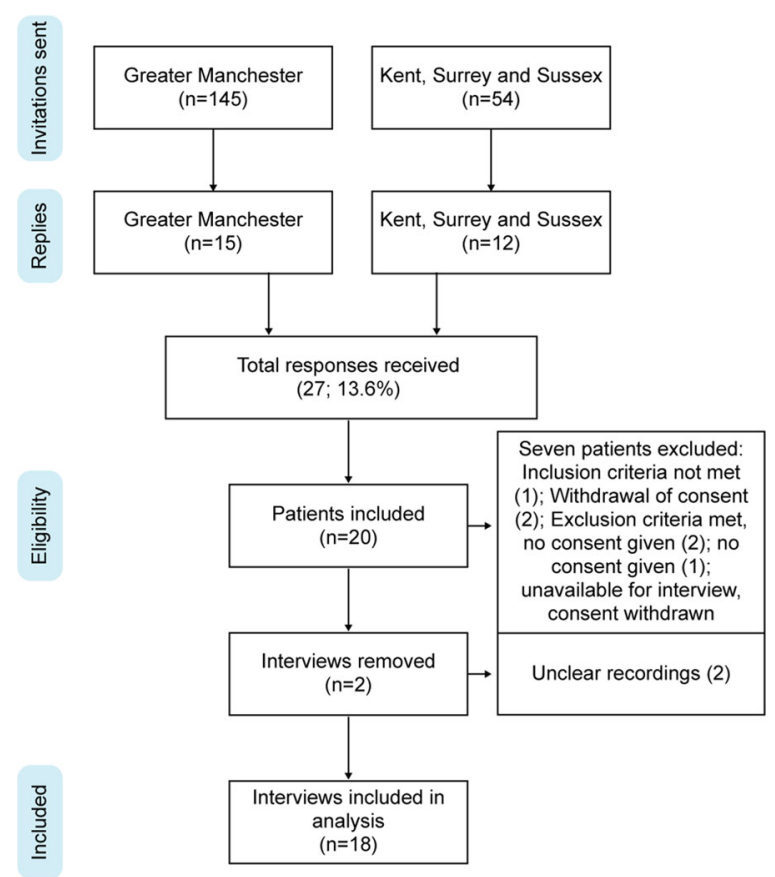

Fig. 1 Summary of patient recruitment and participation

collection and analysis were discontinued when data saturation was achieved (i.e. no new information was discovered) [23]. Identified themes were agreed by all authors.

\section{RESULTS}

\section{Study Size}

Of the 199 invitation packs sent, 27 positive responses were received and 7 respondents were excluded (Fig. 1). A total of 20 interviews were conducted (face-to-face, $n=13$; telephone, $n=7$ ), between June and August 2018. Subsequently, two interviews were excluded because of poor-quality recordings not allowing accurate transcription and analysis. Upon transcribing and analysing the data from the 18 interviews, the study team recognised that saturation had been reached.

\section{Participant Demographics}

Interviews from five men and 13 women, with an overall mean age of 70 years (range 57-80), were included in the final analysis (Table 1). The most common OAB symptoms reported included urgency, frequency, incontinence and nocturia.

\section{Factors Influencing Persistence and/ or Adherence to Prescribed OAB Medication}

Several key themes of factors influencing persistence and/or adherence to prescribed $\mathrm{OAB}$ medication were identified, broadly categorised into four subgroups: patients' attitude and condition adaption behaviour; support with treatment; discontinuing treatment; drug/condition hierarchy (Fig. 2).

\section{Patients' Attitude to Medication and Condition Adaptation Behaviour}

Some participants questioned the necessity of taking medication and balanced the perceived risks (side effects) against the clinical benefits in their decisions to start or continue taking $\mathrm{OAB}$ medication-'If it is necessary to take the medication, then it should be taken as long as there are no noticeable side effects', (participant \#16). Other participants (\#7, 8 and 17) expressed that they would take medication if there were tangible benefits (improvement in OAB symptoms). However, one participant (\#7) would advise others against starting OAB medication, despite experiencing some symptom relief, due to a side effect (headache) that led to their nonadherence.

Negative beliefs regarding risk of side effects were the basis of one participant's (\#13) decision to only take $\mathrm{OAB}$ medication on an 'asneeded' basis, despite a conviction that regular usage would be beneficial, while another participant (\#10) was reluctant to accept a dose increase of $\mathrm{OAB}$ medication, although acknowledging it might improve symptoms. One participant (\#5) chose not to be exposed to any potential danger from side effects/complications, on the basis of a prior hospitalisation following an incorrect prescription for hypertension.

Non-adherence was largely intentional (e.g. participants did not forget to take their 
Table 1 Study participants demographic and background information

\begin{tabular}{lllll}
\hline Area & Participant number & Sex & Age (years) & Main OAB symptoms \\
\hline Greater Manchester & 1 & M & 64 & Urgency, incontinence \\
2 & F & 66 & Urgency, incontinence, nocturia \\
3 & F & 58 & Frequency, urgency, incontinence \\
4 & F & 69 & Urgency, incontinence \\
5 & F & 71 & Frequency, urgency \\
6 & F & 57 & Frequency, nocturia, incontinence \\
7 & F & 70 & Frequency, urgency, high volume \\
8 & F & 77 & Frequency \\
9 & F & 80 & Incontinence, urgency \\
10 & F & 58 & Urgency, nocturia, incontinence \\
11 & F & 78 & Incontinence, urgency, nocturia \\
12 & F & 61 & Frequency, incontinence, \\
Kent, Surrey, and Sussex & & & high volume at night \\
13 & M & 65 & Urgency, frequency \\
15 & M & 78 & Nocturia, urgency \\
16 & F & 80 & Incontinence, nocturia, urgency \\
17 & M & 71 & Urgency, incontinence, nocturia \\
\hline
\end{tabular}

$F$ female, $M$ male, $O A B$ overactive bladder

medication). One participant (\#2) distinguished between their OAB medication, which they had decided not to take, and their other daily medications that they continued to take. Descriptors from participants in reference to remembering to take medication included 'no challenges at all' (participant \#17) and 'automatic' (participant \#8). Strategies for avoiding non-intentional adherence included incorporating medicine taking into a daily routine (e.g. taking tablets with breakfast or just before going to bed); keeping medication in conspicuous places and in the line of sight; and using dosset boxes. Although these strategies were highly effective, they were not without certain challenges e.g. if the usual schedule was disrupted by a change in location or setting. One participant (\#9), who described a routine of taking medication around breakfast time, stated that adherence would be difficult if a new medication was prescribed that needed to be taken at a different time of the day.

Participants modified their daily behaviours and planned ahead in order to foster a sense of safety and confidence, including keeping an acute awareness of toilet locations, using the toilet pre-emptively, and gravitating towards familiar places, such as their own home, where others would not witness or be affected by their symptoms. Among female participants, absorbent pads were commonly used as a precautionary measure. Several participants described variants of 'planning your day around being near a toilet' and 'life around toilets'. This was a 

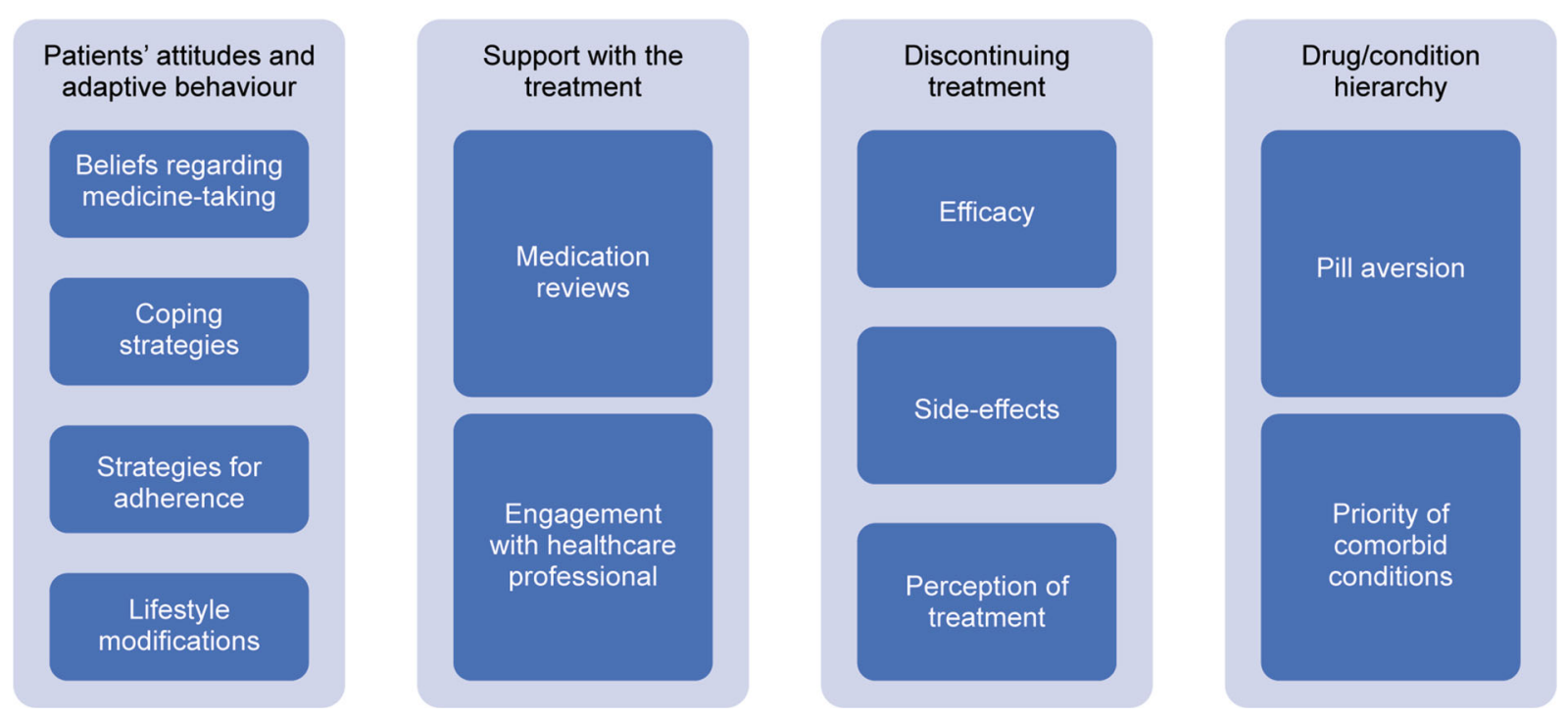

Fig. 2 Identified themes from interviews on persistence and adherence to $O A B$ medication

key element in the pursuit of feeling secure'Sainsbury's have got loos, so... if I need a loo, I'm alright' (participant \#5). One participant (\#7) mentioned planning social interactions specifically in places where they had knowledge of the availability of public toilets. One participant (\#6) described a reluctance to go on holiday-'I haven't been this year, 'til I wanted to sort me bladder out', and being around people they were comfortable with-'In a friend's apartment, I'm safe... they know that I can't control'.

Some participants demonstrated a reluctance to make recommended lifestyle modifications, including two participants (\#12 and 17) who perceived difficulties/impracticalities of incorporating pelvic floor exercises into their routine, and another (\#7) who did not adhere to caffeine avoidance advice, as this entailed giving up a daily habit.

\section{Support with Treatment}

Several participants described a lack of formal reviews for $\mathrm{OAB}$ medication, in contrast to routine medication reviews conducted for their other conditions. Some participants felt their general practitioners (GPs) were 'too busy' to conduct monitoring reviews. Reviews for $\mathrm{OAB}$ were rushed or overlooked, and medicines that caused specific problems were given prominence during the review process, which was focused on problem-solving, rather than optimising their care. In other cases, repeat prescriptions were issued without medication reviews.

One participant (\#16) mentioned that the lack of their own reviews was due to a reluctance to visit the GP, downplaying their own needs against those of others who 'really are ill'. A lack of communication with the HCP, a preference to avoid medication, and a doubted $\mathrm{OAB}$ diagnosis were the reasons given by another participant (\#17) for not attending a repeat visit after initially being prescribed a 1-month supply of OAB medication. One participant (\#13) decided to take OAB medication sporadically to manage their inconsistent symptoms, because the HCP appeared uninterested and did not stress taking the tablets daily. Although convinced that daily medication would offer complete symptom relief, this was tempered by a fear of unknown long-term effects. However, some participants described their GP as being supportive, providing invitations for reviews and imparting confidence.

\section{Unmet Efficacy/Tolerability Expectations}

Over half of participants cited lack of efficacy as a reason for non-adherence and/or non-persistence of $\mathrm{OAB}$ medication (Table 2). One 
Table 2 Reasons for discontinuing OAB medication

\begin{tabular}{|c|c|c|c|c|}
\hline $\begin{array}{l}\text { Participant } \\
\text { number }\end{array}$ & Sex & $\begin{array}{l}\text { Age } \\
\text { (years) }\end{array}$ & $\begin{array}{l}\text { Primary reasons for } \\
\text { discontinuation }\end{array}$ & Other reasons \\
\hline 1 & M & 64 & Lack of efficacy & Tablet being 'nothing special' \\
\hline 2 & $\mathrm{~F}$ & 66 & Side effects & - \\
\hline 3 & $\mathrm{~F}$ & 58 & Did not discontinue & - \\
\hline 4 & $\mathrm{~F}$ & 69 & $\begin{array}{l}\text { Lack of efficacy and requirement } \\
\text { for ECGs }\end{array}$ & Fear of unknown effects \\
\hline 5 & $\mathrm{~F}$ & 71 & Side effects & Taking too many tablets \\
\hline 6 & $\mathrm{~F}$ & 57 & Waning efficacy, side effects & Onabotulinumtoxin $\mathrm{A}$ \\
\hline 7 & $\mathrm{~F}$ & 70 & Side effects & Taking too many tablets \\
\hline 8 & $\mathrm{~F}$ & 77 & Lack of efficacy & Suspected side effect \\
\hline 9 & $\mathrm{~F}$ & 80 & Side effects & Fear of unknown effects \\
\hline 10 & $\mathrm{~F}$ & 58 & Side effects & $\begin{array}{l}\text { Aversion to dose escalation, wanting to switch } \\
\text { medication }\end{array}$ \\
\hline 11 & $\mathrm{~F}$ & 78 & Did not discontinue & - \\
\hline 12 & $\mathrm{~F}$ & 61 & Lack of efficacy, side effects & $\begin{array}{l}\text { Unconvinced of } \mathrm{OAB} \text { diagnosis (more a 'weak } \\
\text { bladder') }\end{array}$ \\
\hline 13 & M & 65 & Fear of side effects & Unclear instructions \\
\hline 14 & M & 78 & Lack of efficacy & Side effects \\
\hline 15 & $\mathrm{~F}$ & 80 & Lack of efficacy, side effects & $\begin{array}{l}\text { Surgery stopped the medication } \\
\text { because of potential cognitive effects }\end{array}$ \\
\hline 16 & $\mathrm{M}$ & 71 & Lack of efficacy, side effects & Trial and error \\
\hline 17 & $\mathrm{~F}$ & 67 & Unclear instructions & Pill aversion \\
\hline 18 & M & 70 & Lack of efficacy & - \\
\hline
\end{tabular}

$E C G$ electrocardiogram, $F$ female, $M$ male, $O A B$ overactive bladder

participant (\#14) expressed frustration due to poor efficacy and the need to take sleeping pills to counteract the secondary effects of next-day fatigue from nocturia. According to many participants, side effects further prompted their decision to discontinue ineffective medication. Reported side effects affecting persistence and/or adherence included dry mouth and headaches. Drowsiness was mentioned by several participants, but mostly in a positive context as an aid to sleep (reducing the effects of nocturia). One participant (\#18), who initially experienced a 'minor improvement' in urinary symptoms from $\alpha$-blocker monotherapy, subsequently received an add-on antimuscarinic agent, but did not perceive any 'great improvement' from combination therapy. Together with the side effect of dry mouth, this led to complete treatment discontinuation; this participant was still symptomatic and waking several times a night. One participant (\#15) experienced a dull stomach ache from a twice-daily antimuscarinic agent, which resulted in once-daily treatment and eventual discontinuation (advised by the HCP) due to potential effects on memory. Subsequently, they were not offered an alternative 
treatment and continued to experience the wax and wane of $\mathrm{OAB}$ symptoms. $\beta_{3}$-adrenoceptor agonists were linked to a fear of future side effects by another participant (\#4) due to additional blood pressure and electrocardiogram monitoring required while on treatment.

Doubtful efficacy together with a suspicion that the treatment was causing a deterioration in eyesight led to one participant (\#12) discontinuing treatment. This perceived lack of efficacy was further reinforced by an assessment of the HCP's demeanour (while initially prescribing the drug), interpreted as being 'unsure whether these tablets worked or not'. The participant subsequently re-evaluated the side effects of the medication, attributing the decline in eyesight to advancing age instead, which coincided with a renewed motivation to control symptoms after taking up golf. They remained adherent despite still doubting the effectiveness of medication. Another participant (\#10) was unsure as to which of their medications were responsible for their symptoms of blurred vision and gastro-oesophageal disturbances and intended to discontinue the $\mathrm{OAB}$ medication to gauge how much of an effect this might have. They also cited a lack of awareness about the availability of a range of medications for $\mathrm{OAB}$ as one of the reasons for not returning to the GP to try a different drug'I think from reading that form [patient information sheet], there appears to be quite a lot of different drugs for, the same problem. And my GP didn't offer me a choice or anything'.

\section{Drug/Condition Hierarchy}

Participants showed a general pill aversion, choosing to discontinue $\mathrm{OAB}$ medication while prioritising other conditions. For example, one participant (\#7) had discontinued several OAB medications because of side effects and a wish to avoid tablets where possible, while maintaining adherence to hypertension medication and letrozole, on the basis of the potentially serious consequences of not doing so. This was echoed by participant \#16 who described adherence to hypertension medication: 'Oh I take those without fail, because my blood pressure was quite high'. Some participants chose to delay $O A B$ treatment until they could no longer endure the symptoms and associated consequences. One participant (\#10) became more open to treatment following a worsening of $\mathrm{OAB}$, due to the effect of their $\mathrm{OAB}$ symptoms on chronic migraines. Conversely, the presence of comorbidities, including liver disease culminating in a liver transplant, was a driving factor in adherence to OAB medication for one participant (\#1), who persisted with treatment despite lack of efficacy; now on their third OAB medication (an antimuscarinic agent), they were planning to request a fourth. Nevertheless, they were reluctant to seek HCP advice specifically for OAB (despite worsening of symptoms) and were delaying consultation until another healthcare need arose.

When participants did consult their HCP about their $\mathrm{OAB}$, they often felt that the condition was regarded with low priority by the HCP. According to one participant (\#13), the HCP set the tone of low importance of $\mathrm{OAB}$ and its treatment by giving the impression of providing a diagnosis and treatment to 'get rid' of the patient when an ultrasound test did not reveal any particular issue. This was compounded by there being no review of OAB medication, with the HCP supplying prescriptions only. One participant (\#3), who worked in a GP clinic, drew a comparison between the relative prominence given to conditions such as asthma and prostate cancer against the dearth of information publicly available for 'day-to-day' conditions, such as $\mathrm{OAB}$, which 'really interfere with your lifestyle'. This was mirrored by another participant's (\#12) opinion that there is a lack of effort into developing $\mathrm{OAB}$ medication, as researchers focused on the 'big things'.

\section{DISCUSSION}

Non-adherence and non-persistence with treatment are behaviours that feature prominently among patients with $\mathrm{OAB}[24,25]$. Through qualitative patient interviews, this study provides insights into the complex and individualistic factors driving such behaviours in patients who have undergone pharmacological treatment for $\mathrm{OAB}$.

The interviews uncovered several common themes surrounding non-adherence and/or 
non-persistence of $\mathrm{OAB}$ medication. Failure to take $\mathrm{OAB}$ medication was largely intentional, with many participants making decisions on the basis of a perceived balance of benefits and risks and/or lifestyle choices. Unintentional non-adherence was not identified as a problem, as participants tended to alter their behaviour to facilitate medicine taking. In this study, the main reasons for treatment discontinuation, as reported by participants, were, firstly, a perceived lack of efficacy, resulting in unmet treatment expectations and, secondly, experience of side effects (or fear of potential side effects). There was also a general aversion to taking chronic medication, as well as a tendency to prioritise medications on the basis of the perceived prominence of the condition and the consequences of not taking the medication, giving rise to a drug/condition hierarchy. Lack of engagement and support from HCPs were also factors that may have contributed to poor treatment persistence for OAB.

Dry mouth was the most widely mentioned side effect leading to discontinuation of $\mathrm{OAB}$ medication, followed by headache and nausea-effects typically associated with antimuscarinic therapy and commonly cited as reasons for treatment discontinuation [26, 27]. Improved rates of persistence and/or adherence have been reported with the $\beta_{3}$-adrenoceptor agonist mirabegron compared with antimuscarinic agents [16, 28, 29], perhaps owing to lower rates of typical antimuscarinic adverse events [14]. Many participants were receiving multiple medications for comorbidities, but sometimes incorrectly attributed other undesirable effects to their $\mathrm{OAB}$ medication. The choice to discontinue the $\mathrm{OAB}$ medication over other treatments may suggest a poor perception of the benefit-risk balance of available $O A B$ treatment options, as well as assigning a low priority to managing $\mathrm{OAB}$.

The findings of the current study are aligned with the results of a survey from 5392 respondents using antimuscarinic agents for $\mathrm{OAB}$ in the USA [18], among whom 1322 (24.5\%) reported discontinuing one or more antimuscarinic medications during the previous 12 months. The most commonly reported reasons for discontinuation were that 'it didn't work as expected' (46.2\%), 'switched to a new medication' (25.1\%), 'learned to get by without medication' (23.3\%), and 'I had side effects' (21.1\%). A proportion of participants (11\%) indicated a general aversion to taking any medication. In another US survey of OAB, among the 138 women who reported that they had taken or were currently taking OAB medication, half of them had discontinued $\mathrm{OAB}$ medication at some point; the most common reasons were inadequate efficacy $(42 \%)$ and adverse events/intolerability (30\%) [30].

The issue of drug/condition hierarchy was evident in several participants who opted to discontinue $\mathrm{OAB}$ treatment while prioritising other conditions, such as hypertension, for which the consequences of stopping treatment would be more drastic in their opinion. This verifies the findings of other studies showing that rates of persistence and adherence appear to be particularly low for $\mathrm{OAB}$ medications (specifically antimuscarinic agents) compared with other chronic conditions, such as diabetes, glaucoma, hyperlipidaemia, osteoporosis and hypertension [25].

Better persistence and/or adherence to $\mathrm{OAB}$ medication is associated with improved urinary symptoms and HRQoL $[19,20]$. OAB management primarily through coping strategies in preference to HCP consultation has been highlighted in several studies $[9,13,31]$. Similarly, in the current study, some participants downplayed or normalised their symptoms and 'learned to live with it' by employing coping strategies-these may have a negative impact on patients' HRQoL and may also hinder the realisation of potential clinical benefits from $\mathrm{OAB}$ treatment. This was exemplified by one participant who had resumed OAB medication in order to pursue a new hobby.

In the current study, barriers to patients seeking initial help included a perceived lack of publicly displayed information on $\mathrm{OAB}$, as well as a general unawareness of the availability of treatments. More information may help patients recognise how widespread such symptoms are, leading to less embarrassment in discussing them and thereby facilitating helpseeking behaviour. This may also help patients to decide to pursue better care for their $\mathrm{OAB}$, 
rather than incidentally reporting issues while visiting the GP for other health concerns. Raising awareness to the multiple medications available for $\mathrm{OAB}$ may also provide motivation to pursue alternative treatment. The progressive use of coping strategies may also prevent patients from presenting to their HCP by masking the extent of their condition until the psychological burden becomes too great or they have a significant event, such as an incontinence episode in public.

The finding that many participants discontinued treatment because of unmet efficacy/tolerability expectations suggests that HCPs might be able to encourage persistence and/or adherence by fostering realistic expectations about the onset of efficacy and the extent of benefits $[18,30]$. This could include educating patients that side effects of medication may manifest before improvements in symptoms become apparent, and discussing how side effects could be managed. Furthermore, realistic treatment goals could focus on practical factors that are limiting the patient's HRQoL, such as attending more social events or travelling longer distances, rather than simply focusing on symptoms.

The demeanour of HCPs during consultations led to participants harbouring doubts of both the diagnosis and the treatments offered. The perceived disinterest of HCPs to review OAB medication, alongside participants being reluctant to chase such reviews, has left a gap in patient care, leading to poor treatment persistence. HCPs can support treatment persistence and/or adherence through: the use of patient-centred communication with more discussion of critical topics (e.g. the impact of $\mathrm{OAB}$ on $\mathrm{HRQ}$ L , and concerns about treatment) [32]; involving patients in treatment decisions; reviewing medicines; communication with other HCPs involved in the patient's care [33]. Treatment 'cycling' did not appear to be widespread in the current study (participant \#1 received three $\mathrm{OAB}$ medications and was planning to start a fourth), but as patients may need to try different treatments to find a suitable one, regularly scheduled reviews would allow patients to provide feedback and help optimise their care. Such reviews could be conducted by nurses or pharmacists (e.g. the Medicine Use Review service), thereby relieving pressure on GPs.
There were a number of strengths associated with the current study. Interviews were openended and designed to encourage participants to respond fully in their own words (enabling the generation of rich information). Additionally, data were collected from participants living in two regions, in the North West and in the South of England. The study also had a number of limitations: invited individuals were preselected by HCPs, thus selecting only those who had sought healthcare for their OAB (selection bias); the study was restricted to English language speakers only; socio-economic factors were not considered; the duration of treatment for OAB medications was not captured; and the type of medication taken for other conditions was not formally collected and, therefore, anticholinergic burden may have been a factor influencing $\mathrm{OAB}$ medication-taking behaviour.

In addition to no improvement or deterioration of health, non-adherence and/or non-persistence may lead to higher economic costs with respect to wasted medicines and increased demands for other healthcare resources; economic considerations of poor persistence and/or adherence in $\mathrm{OAB}$ could be a feature of future research. Additional data collection methods (e.g. focus groups, questionnaires/surveys), which may provide a more complete picture, could be employed in future studies. Further work is needed to investigate the extent to which previously reported determinants of persistence and/or adherence, such as female (sex), older age group, use of extended-release formulations and treatment experience [14], influence OAB medication persistence and/or adherence. These could take the form of longitudinal studies following a patient's journey through medication taking to understand their decision-making processes better. Drug/condition hierarchy and its effect on disease management and outcomes is also a potential area of interest for further investigation. This is particularly relevant in $\mathrm{OAB}$, where patients may have other long-term comorbidities.

\section{CONCLUSION}

In the current study, the primary reason for non-adherence and/or discontinuing $\mathrm{OAB}$ 
medication, as reported by patients, was perceived poor efficacy/unmet expectations, followed by side effects. Patients also showed an aversion to long-term medication taking and opted to discontinue $\mathrm{OAB}$ treatment, according low priority to $\mathrm{OAB}$ relative to other comorbidities. By providing confident diagnoses, managing expectations of treatment benefit, educating patients on managing side effects, and raising awareness of the availability of multiple treatments, HCPs may be able to support more adherent and persistent behaviours among patients with OAB.

\section{ACKNOWLEDGEMENTS}

The authors would like to thank study participants, Greater Manchester and Kent, Surrey, and Sussex Research and Development sites, as well as participating Primary Care Practices for their involvement and assistance with this study.

Funding. This study was co-funded by Innovate UK and Astellas Pharma Europe Ltd (APEL), as part of a knowledge transfer partnership (KTP) between Manchester Metropolitan University and APEL. The KTP involved a collaboration between Manchester Metropolitan University and APEL who had joint roles in: the design and conduct of the study; management, analysis and interpretation of the data; preparation, review and approval of the manuscript. The journal's Rapid Service and Open Access Fees were funded by APEL.

Medical Writing Assistance. Medical writing support in the preparation of this article was provided by Shamika de Silva, $\mathrm{PhD}$ and Tyrone Daniel, PhD of Bioscript Medical; this was funded by APEL.

Authorship. All named authors meet the International Committee of Medical Journal Editors (ICMJE) criteria for authorship for this article, take responsibility for the integrity of the work as a whole, and have given their approval for this version to be published.
Disclosures. Margarita Landeira is a current employee of APEL. Patrick Covernton is a current employee of APEL. Jameel Nazir was an employee of APEL at the time the study was conducted and is now an employee of Sobi. Francis Fatoye was a member of the KTP team, which received research grants from APEL. Maxine Holt was a member of the KTP team, which received research grants from APEL. Sarah Grogan was a member of the KTP team, which received research grants from APEL. Sue Powell was a member of the KTP team, which received research grants from APEL. Mahmood Ali completed secondments at APEL. Ashley Jaggi completed secondments at APEL. Leanne Staniford has nothing to disclose.

Compliance with Ethics Guidelines. This study has gone through a health research authority and ethics review with NW Liverpool East REC (Central Manchester is the lead Research \& Development site). IRAS project ID 198502. All participants provided their written consent to take part in this study.

Data Availability. All data generated or analysed during this study are included in this published article or as supplementary information files. Access to anonymised individual participant level data will not be provided for this trial as it meets one or more of the exceptions described on http://www. clinicalstudydatarequest.com under 'Sponsor Specific Details for Astellas'.

Open Access. This article is distributed under the terms of the Creative Commons Attribution-NonCommercial 4.0 International License (http://creativecommons.org/licenses/ by-nc/4.0/), which permits any noncommercial use, distribution, and reproduction in any medium, provided you give appropriate credit to the original author(s) and the source, provide a link to the Creative Commons license, and indicate if changes were made. 


\section{REFERENCES}

1. D'Ancona C, Haylen B, Oelke $\mathrm{M}$, et al. The International Continence Society (ICS) report on the terminology for adult male lower urinary tract and pelvic floor symptoms and dysfunction. Neurourol Urodyn. 2019;38(2):433-77.

2. Irwin DE, Kopp ZS, Agatep B, Milsom I, Abrams P. Worldwide prevalence estimates of lower urinary tract symptoms, overactive bladder, urinary incontinence and bladder outlet obstruction. BJU Int. 2011;108(7):1132-8.

3. Irwin DE, Milsom I, Hunskaar S, et al. Populationbased survey of urinary incontinence, overactive bladder, and other lower urinary tract symptoms in five countries: results of the EPIC study. Eur Urol. 2006;50(6):1306-14.

4. Eapen RS, Radomski SB. Gender differences in overactive bladder. Can J Urol. 2016;23(Suppl 1):2-9.

5. Wennberg AL, Molander U, Fall M, Edlund C, Peeker R, Milsom I. A longitudinal population-based survey of urinary incontinence, overactive bladder, and other lower urinary tract symptoms in women. Eur Urol. 2009;55(4):783-91.

6. Office for National Statistics. Overview of the UK population: November 2018. London: ONS; 2018.

7. Coyne KS, Sexton CC, Irwin DE, Kopp ZS, Kelleher CJ, Milsom I. The impact of overactive bladder, incontinence and other lower urinary tract symptoms on quality of life, work productivity, sexuality and emotional well-being in men and women: results from the EPIC study. BJU Int. 2008;101(11):1388-95.

8. Irwin DE, Milsom I, Kopp Z, Abrams P, Cardozo L. Impact of overactive bladder symptoms on employment, social interactions and emotional well-being in six European countries. BJU Int. 2006;97(1):96-100.

9. Nicolson P, Kopp Z, Chapple CR, Kelleher C. It's just the worry about not being able to control it! A qualitative study of living with overactive bladder. Br J Health Psychol. 2008;13(Pt 2):343-59.

10. European Association of Urology. Urinary incontinence. 2019. https://uroweb.org/guideline/urinaryincontinence/\#4. Accessed 10 Apr 2019.

11. Keam SJ. Vibegron: first global approval. Drugs. 2018;78(17):1835-9.

12. Kim TH, Lee KS. Persistence and compliance with medication management in the treatment of overactive bladder. Investig Clin Urol. 2016;57(2):84-93.

13. Ricci JA, Baggish JS, Hunt TL, et al. Coping strategies and health care-seeking behavior in a US national sample of adults with symptoms suggestive of overactive bladder. Clin Ther. 2001;23(8):1245-59.

14. Yeowell G, Smith P, Nazir J, Hakimi Z, Siddiqui E, Fatoye F. Real-world persistence and adherence to oral antimuscarinics and mirabegron in patients with overactive bladder $(\mathrm{OAB})$ : a systematic literature review. BMJ Open. 2018;8(11):e021889.

15. Krhut J, Gartner M, Petzel M, et al. Persistence with first line anticholinergic medication in treatmentnaive overactive bladder patients. Scand J Urol. 2014;48(1):79-83.

16. Chapple CR, Nazir J, Hakimi Z, et al. Persistence and adherence with mirabegron versus antimuscarinic agents in patients with overactive bladder: a retrospective observational study in UK clinical practice. Eur Urol. 2017;72(3):389-99.

17. World Health Organization. Adherence to longterm therapies: evidence for action. 2003. http:// apps.who.int/iris/bitstream/handle/10665/42682/ 9241545992.pdf;jsessionid=2B089E9D194432FE6 E2425E15D5B6943? sequence $=1$. Accessed 7 Feb 2019.

18. Benner JS, Nichol MB, Rovner ES, et al. Patient-reported reasons for discontinuing overactive bladder medication. BJU Int. 2010;105(9):1276-82.

19. Kim TH, Choo MS, Kim YJ, Koh H, Lee KS. Drug persistence and compliance affect patient-reported outcomes in overactive bladder syndrome. Qual Life Res. 2016;25(8):2021-9.

20. Andy UU, Arya LA, Smith AL, et al. Is self-reported adherence associated with clinical outcomes in women treated with anticholinergic medication for overactive bladder? Neurourol Urodyn. 2016;35(6):738-42.

21. Balkrishnan R, Bhosle MJ, Camacho FT, Anderson RT. Predictors of medication adherence and associated health care costs in an older population with overactive bladder syndrome: a longitudinal cohort study. J Urol. 2006;175(3 Pt 1):1067-71.

22. Braun V, Clarke V. Using thematic analysis in psychology. Qual Res Psychol. 2006;3(2):77-101.

23. Faulkner SL, Trotter SP. Data saturation. The international encyclopedia of communication research methods. 2017:1-2. https://onlinelibrary.wiley. com/doi/abs/10.1002/9781118901731.iecrm0060. Accessed 1 May 2019. 
24. Cutler RL, Fernandez-Llimos F, Frommer M, Benrimoj C, Garcia-Cardenas V. Economic impact of medication non-adherence by disease groups: a systematic review. BMJ Open. 2018;8(1):e016982.

25. Yeaw J, Benner JS, Walt JG, Sian S, Smith DB. Comparing adherence and persistence across 6 chronic medication classes. J Manag Care Pharm. 2009;15(9):728-40.

26. Sexton CC, Notte SM, Maroulis C, et al. Persistence and adherence in the treatment of overactive bladder syndrome with anticholinergic therapy: a systematic review of the literature. Int J Clin Pract. 2011;65(5):567-85.

27. Vouri SM, Kebodeaux CD, Stranges PM, Teshome BF. Adverse events and treatment discontinuations of antimuscarinics for the treatment of overactive bladder in older adults: a systematic review and metaanalysis. Arch Gerontol Geriatr. 2017;69:77-96.

28. Wagg A, Franks B, Ramos B, Berner T. Persistence and adherence with the new beta- 3 receptor agonist, mirabegron, versus antimuscarinics in overactive bladder: early experience in Canada. Can Urol Assoc J. 2015;9(9-10):343-50.

29. Wagg AS, Foley S, Peters J, Nazir J, Kool-Houweling L, Scrine L. Persistence and adherence with mirabegron vs antimuscarinics in overactive bladder: retrospective analysis of a UK general practice prescription database. Int J Clin Pract. 2017; 71(10):e12996.

30. Diokno AC, Sand PK, Macdiarmid S, Shah R, Armstrong RB. Perceptions and behaviours of women with bladder control problems. Fam Pract. 2006;23(5):568-77.

31. Blasco P, Valdivia MI, Ona MR, Roset M, Mora AM, Hernandez M. Clinical characteristics, beliefs, and coping strategies among older patients with overactive bladder. Neurourol Urodyn. 2017;36(3):774-9.

32. Hahn SR, Bradt P, Hewett KA, Ng DB. Physicianpatient communication about overactive bladder: results of an observational sociolinguistic study. PLoS One. 2017;12(11):e0186122.

33. National Institute for Health and Care Excellence. Medicines adherence: involving patients in decisions about prescribed medicines and supporting adherence; clinical guideline [CG76]. 2009. https://www. nice.org.uk/guidance/cg76/chapter/1-Guidance\#sup porting-adherence. Accessed 7 Mar 2019. 